\title{
Treatment of Cutaneous Lymphoid Hyperplasia with Rituximab: A Case Report of 3 Patients
}

\author{
Marzieh Matin ${ }^{1}$, Ali Asilian ${ }^{1}$, Samaneh Mozafar Poor ${ }^{1}$, Elaheh Haftbaradaran ${ }^{{ }^{*}}$ and Amir Feily ${ }^{2}$ \\ ${ }^{1}$ Department of Dermatology, Isfahan University of Medical Sciences, Isfahan, Iran \\ ${ }^{2}$ Skin and Stem Cell Research Center, Tehran University of Medical Sciences, Tehran, Iran
}

"Corresponding author: Elaheh Haftbaradaran, Skin Diseases and Leishmaniasis Research Center, Isfahan University of Medical Sciences, Isfahan, Iran, E-mail: elahe_md2003@yahoo.com

Received date: February 17, 2019; Accepted date: March 14, 2019; Published date: March 22, 2019

Copyright: (c) 2019 Matin M, et al. This is an open-access article distributed under the terms of the Creative Commons Attribution License, which permits unrestricted use, distribution, and reproduction in any medium, provided the original author and source are credited.

\begin{abstract}
Benign Cutaneous Lymphoid Hyperplasia (CLH) is a B-cell pseudolymphoma of an unknown origin. The face is the most affected site in CLH. This report presents the cases of 3 patients with CLH who were treated with rituximab.

It is a hypothesis that rituximab is useful for treating resistant-to-relapsing forms of CLH that do not respond to previous therapies but must be checked with appropriate designs research.
\end{abstract}

Keywords: Cutaneous lymphoid hyperplasia; Rituximab; Erythematous papules

\section{Case Report}

Cutaneous Lymphoid Hyperplasia (CLH) is a benign lymphoid reactive process resulting from various antigenic stimuli and may progress to overt lymphoma [1]. Patients with CLH typically present with macules or papules on the face with or without any recipitating cause. CLH is diagnosed by performing biopsies of facial lesions. CLH usually regresses after removing the offending agent. In some cases, intralesional corticosteroid injections or radiotherapy is used [2]. In this study, we used intralesional rituximab therapy for treating 3 patients with CLH.

\section{Patient Presentation}

Case 1: A 41-year-old white woman presented with a 7-month history of itchy papules and erythematous plaques on the face. During this period, she was treated using intralesional injections of corticosteroids and showed relative improvement. However, her treatment was stopped because of atrophy induced by corticosteroid injections. She did not have a history of any other disease (Figure 1A).

Physical examination detected erythematous papules and plaques on the cheeks and sides of the face. She did not have lymphadenopathy and organomegaly and yielded normal results for other laboratory tests.

Skin biopsy of the lesions was performed. Histopathological analysis of the biopsied skin samples showed dense infiltration of lymphocytes into the dermis surrounding the vessels and adnexa. In addition, follicles with germinal centers were observed. These findings indicated the presence of CLH (Figure 1B and 1C).
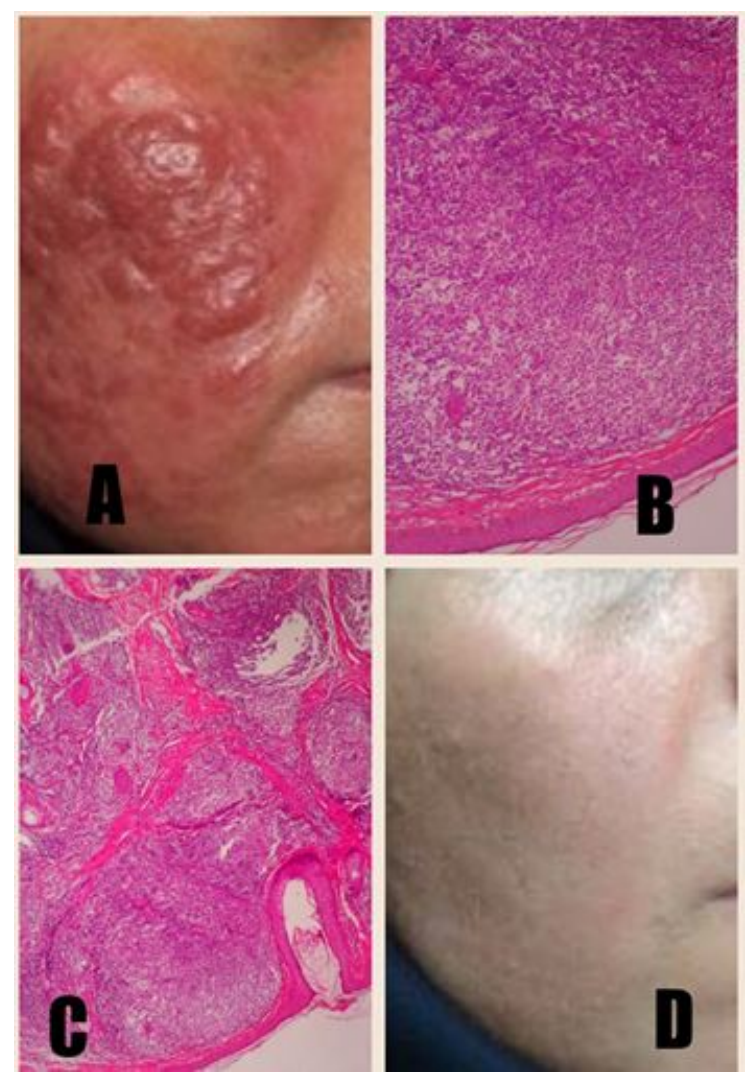

Figure 1: Patient with itchy papules and erythematous plaques on the face treated with intralesional rituximab (10 $\mathrm{mg} / \mathrm{lesion})$ per week for 18 weeks.

The patient was treated with intralesional rituximab $(10 \mathrm{mg} / \mathrm{lesion})$ per week for 18 weeks. The patient developed side effect associated 
with rituximab, such as severe pain during injection, dizziness, hair loss, and forgetfulness. The patient showed complete remission after the treatment; however, recurrence was not observed at 6-month evaluation (Figure $1 \mathrm{D})$.

Case 2: A 65-year-old woman with multiple erythematous papules on the arm, which appeared approximately a month previously and for which no treatment was administered, was referred to Al-Zahra Medical Center. The patient had a history of high blood pressure for 10 years and was treated with amlodipine and sotalol during this period (Figure 2A).

Physical examination detected multiple stiff erythematous papules on the arm. The patient did not have lymphadenopathy and organomegaly and yielded normal results for other laboratory tests.

Skin biopsy of the lesions was performed. Histopathology analysis of the biopsied samples showed diffuse infiltration of basophils and lymphocytes into the dermis. Immunostaining detected CD20+ and $\mathrm{CD} 3+$ lymphocytes in the germinal centers of the follicles, indicating the presence of CLH (Figure 2B and 2C).

The patient was treated with intralesional rituximab (10 mg/lesion) per week for 6 weeks. She experienced rituximab-associated side effects such as pain during injection. Her lesions were treated completely, and no recurrence was observed at 6-month evaluation (Figure 2D).
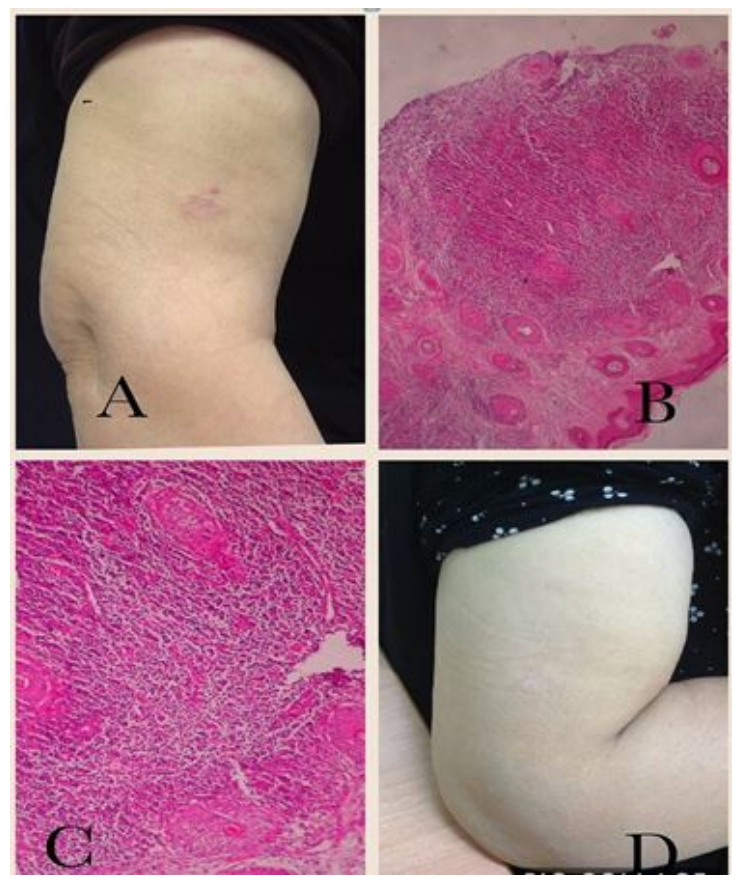

Figure 2: Patient with multiple erythematous papules on the arm treated with intralesional rituximab $(10 \mathrm{mg} / \mathrm{lesion})$ per week for 6 weeks.

Case 3: A 62-year-old woman with a 2-year history of itchy erythematous papules and plaques was treated using corticosteroids and oral antihistamines. However, this treatment did not lead to recovery, and the number of lesions increased. The lesions present on the ear progressed to the cheek and forehead.
The patient had undergone pancreatic surgery approximately 10 years previously and received chemotherapy and radiotherapy for pancreatic cancer, which resulted in improvement and no side effects (Figure 3A).

Physical examination detected multiple erythematous papules and plaques on the forehead, sides of the face, and the cheeks. The patient did not have lymphadenopathy and organomegaly and did not yield any other abnormal results. Furthermore, the patient yielded normal results for laboratory tests. Analysis of biopsied skin samples indicated CLH.

The patient was treated with intralesional rituximab ( $10 \mathrm{mg} / \mathrm{lesion})$ per week for 18 weeks. Rituximab infusion induced side effects such as severe pain during injection, dizziness, headache, lethargy, limb pain, and nausea, which decreased after oral antihistamine treatment (Figure $3 \mathrm{~B})$. However, the patient showed recurrence of the papules and plaques after 1 month.

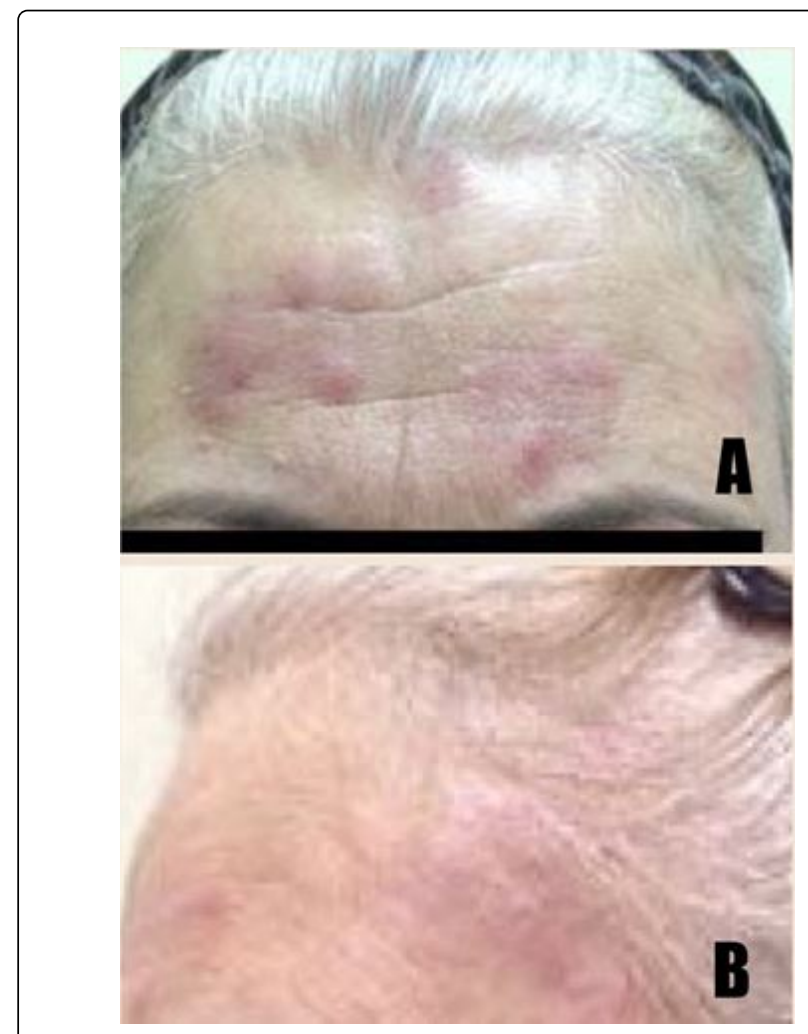

Figure 3: Patient with multiple erythematous papules and plaques on the forehead, sides of the face and the cheeks treated with intralesional rituximab (10 mg/lesion) per week for 18 weeks.

\section{Discussion}

CLH is a lymphoproliferative disorder characterized by the infiltration of polyclonal and polymorphic $\mathrm{T}$ and $\mathrm{B}$ lymphocytes [3-5]. After the identification of its cause, the initial treatment of CLH includes the elimination of the triggering factor. Persistent skin lesions show malignant potential. Different treatments of these lesions with different levels of success are Antibiotics (oral doxycycline), Biological agents (interferon-alfa-2b and TNF-antibodies), Corticosteroids (topical, intralesional, and systemic), External beam radiation, 
Citation: Matin M, Ali Asilian A, Poor SM, Haftbaradaran E, Feily A (2019) Treatment of Cutaneous Lymphoid Hyperplasia with Rituximab: A Case Report of 3 Patients. Dermatol Case Rep 4:150.

Page 3 of 3

Immunosuppressants (azathioprine and cyclosporine) and Ultraviolet B phototherapy [5-10].

Rituximab, a monoclonal antibody that targets the CD20 marker on B cells, became commercially available in 1998 [11]. This drug is currently approved for treating non-Hodgkin lymphoma and rheumatoid arthritis and is well tolerated [3]. The most common adverse effect associated with the systemic administration of rituximab is flushes and moderate pain during local injection [11-13]. Recent studies involving small sample size have used systemic administration (375 $\mathrm{mg} / \mathrm{m}^{2}$ per week for 4 weeks) or intralesional injection of rituximab (10-30 mg/lesion 3 times a week for 4 weeks) for treating CD20 PCBCL and have reported no obvious improvement with the latter mode of treatments $[11,13,14]$. Stephanie et al. used the intralesional injection of rituximab $(10 \mathrm{mg} / \mathrm{ml})$ per week for 18 weeks. This dose was well tolerated, with no side effects. Despite the improvement, relapse was observed gradually after 2 months for erythematous lesions and treatment was changed to $0.1 \%$ tacrolimus, which resulted in significant improvement after 4 weeks. Intralesional rituximab therapy seems to be an appropriate remedy for treating refractory and recurrent $\mathrm{CD} 20+$ lesions that do not respond to previous therapies [15].

\section{Conclusion}

$\mathrm{NEH}$ is a neutrophilic dermatosis characterized by neutrophilic infiltrates within and around eccrine glands. It should be considered in the differential diagnosis of eroded, erythematous plaques, especially in patients receiving high-dose chemotherapy for primary CNS lymphoma. A diagnosis of NEH should be based on histological findings to avoid unnecessary use of antibiotics and changes in chemotherapy regimens.

\section{References}

1. Jeffrey NL, Yang X, Sunghun C, Leonard CS (2007) Cutaneous Lymphoid Hyperplasia: A case report and brief review of the literature. Cutis 79: 445-448.
2. Khan AQ, Tolat T, Patil A (2015) Cutaneous Lymphoid Hyperplasia presenting as bilateral post-auricular swellings: A case report. IJSR 4: 2366-2368.

3. Gilliam AC, Wood GS (2000) Cutaneous lymphoid hyperplasias. Semin Cutan Med Surg 19: 133-141.

4. Dadban A, Truchetet F (2008) Cutaneous lymphoid hyperplasia on a preexistent melanocytic nevus. Dermatology 217: 199-200.

5. Witzig TE, Inwards DJ, Habermann TM (2007) Treatment of benign orbital pseudolymphomas with the monoclonal anti-CD20 antibody rituximab. Mayo Clin Proc 82: 692-699.

6. Anandasabapathy N, Pulitzer M, Epstein W (2008) Pseudolymphoma evolving into diffuse large B-cell lymphoma. Dermatol Online J 14: 22.

7. Taylor RB, Fortney JA, Pollack RB (2010) Radiation therapy for B-cell cutaneous lymphoid hyperplasia. Jpn J Radiol 28: 385-387.

8. Tomar S, Stoll HL, Grassi MA (2009) Treatment of cutaneous pseudolymphoma with interferon alfa-2b. J Am Acad Dermatol 60: 172-174.

9. Benchikhi H, Bodemer C, Fraitag S (1999) Treatment of cutaneous lymphoid hyperplasia with thalidomide: Report of two cases. J Am Acad Dermatol 40: 1005-1007.

10. Guis S, Schiano JM, Bonnet N (2008) Cutaneous pseudolymphoma associated with a TNF-alpha inhibitor treatment: Etanercept. Eur J Dermatol 18: 474-476.

11. Valencak J, Weihsengruber F, Rappersberger K (2009) Rituximab monotherapy for primary cutaneous B-cell lymphoma: Response and follow-up in 16 patients. Ann Oncol 20: 326-330.

12. Good LM, MillerMD, HighWA (2011) Intralesional agents in the management of cutaneous malignancy: A review. J Am Acad Dermatol 64: 413-422.

13. Paul T, Radny P, Kröber SM (2001) Intralesional rituximab for cutaneous B-cell lymphoma. Br J Dermatol 144: 1239-1243.

14. Luger T, Paul C (2007) Potential new indications of topical calcineurin inhibitors. Dermatology 215: 45-54.

15. Stephanie J M, Madeleine D (2011) Treatment of cutaneous lymphoid hyperplasia with the monoclonal anti-CD20 antibody rituximab. 11: 286-288. 